\title{
MODELING PUBLIC TRANSPORT NETWORK SYSTEM BY USING STATISTICS, NETWORK THEORY AND ANT COLONY OPTIMIZATION
}

\author{
Matej BABIČ \\ Faculty of Information Studies \\ Novo mesto, Slovenia \\ e-mail: babicster@gmail.com
}

\section{Ladislav HLUCHÝ}

Institute of Informatics, Slovak Academy of Sciences

Dúbravská cesta 9

84507 Bratislava, Slovakia

e-mail: Ladislav.Hluchy@savba.sk

\section{Šter BRANKO}

Faculty of Computer and Information Science, University of Ljubljana Slovenia

e-mail: branko.ster@fri.uni-lj.si

\section{Janez PovH}

Faculty of Mechanical Engineering, University of Ljubljana

Slovenia

\&

Development Center

Novo mesto, Slovenia

e-mail: janez.povh@fs.uni-lj.si 
Abstract. In some countries, bicycles are often used to access public transit stations, but the proportion of out-of-the-way travel is much smaller due to the limited availability of bicycles. Public bicycles are innovative rental or free bicycle schemes in urban areas that can be used for day-to-day mobility as one-way use is possible and can be considered as part of a public transport system. Different from traditional, mostly leisure bike rental services, they provide fast and easy access and have a variety of organizational layout, business models and useful technology for smart bikes (rented via smart cards or mobile phones). We find that bicyclesharing systems that complement the traditional public transport system could potentially increase the competitiveness and attractiveness of sustainable modes of urban transport and thus help cities to promote sustainable daily mobility. Finally, we emphasize that the availability of open sources of urban transport information, such as public transport in our case, is crucial for analyzing urban mobility patterns. The aim of the research is to analyze and model PPP bicycle rentals using mathematical and computer methods. The article presents the application of the statistical and topological properties of bicycle rental and return network theory in city Novo mesto. The article uses swarm intelligence, a colony of ants to optimize the development of wheels across 14 stations. The wider city Novo mesto region with a population of almost 30000 people, as a key industrial center, is heavily dependent on urban transport.

Keywords: Trasport system, ant colony, network theory

\section{INTRODUCTION}

It is essential that we constantly monitor the use, acceptance and quality of the public bicycle system. Marketing should also aim to encourage people who discovered urban cycling through a public cycling scheme to get their bike and use it daily or for leisure activities. This can help to increase the share of urban cycling in general [1. A key element for successful long-term operation is the development of a well-planned financing strategy. In many cases, startup resources from the public side may be available, but there is little thought about what will happen after this stage. Individual strategies need to be found for each type of scheme. Usage data and critical feedback help optimize bicycle distribution and ensure good availability and quality for users. High acceptance, even among the general public and in use, is a good argument for maintaining the system in the long term. Following a well-established public cycling scheme, the interest of potential users can be reduced. Therefore, it is crucial to remind people of the benefits of the system and to encourage its use in order to reach a stable or growing number of users. The hilly topography throughout downtown can be a barrier to deployment, but can be eliminated by using wheels with additional electric propulsion. Climate does not seem to play such an important role, as successful programs have been implemented under different climatic conditions. Creating favorable frame- 
work conditions for urban cycling public bicycles can be an open door to encourage urban cycling. However, people only use a bicycle if it is safe, convenient and the fast way to travel. Therefore, only cities with a minimum and safe cycling infrastructure, and an integrated cycling promotion strategy fulfill the good framework conditions for the implementation of the public bicycle scheme. This includes measures such as traffic calming, the establishment of a cycling network and secure parking, information, marketing and education [2. Information and communication technology [3] is changing the way cities organize policy making and urban growth. Smart cities [4] base their strategy on the use of information and communication technologies in several fields, such as economy, environment, mobility and governance to transform urban infrastructure and services. Statistics [5] is a form of mathematical analysis that uses quantified models, presentations for a given set of experimental data or real studies. Statistics examine methodologies for collecting, reviewing, analyzing, and inferring data. Metaheuristic solution of combinatorial optimization problems is a modern and fast growing research field [6]. This is mainly due to the importance of combinatorial optimization problems in both science and industry. Ant colony [7] is the most successful metaheuristic method for solving discrete optimization problems, which belongs to the family of swarm intelligence algorithms. It derives the idea from the involvement and behavior of real ants in their search for food. Ant colony optimization is divided into several classes: Ant System (AS), Elitist Ant System (EAS), Rank-Based Ant System (ASrank), Min-Max Ant System (MMAS), and Ant Colony System (ACS). The aim of the research is to analyze public-private partnership bicycle rentals using mathematical and computer methods. The goal of this research is to show where to put the additional six stations at the request of the Novo mesto municipality.

\section{METHODOLOGY}

In 8 authors have explored the influence of different attributes on the choice of cycle path, such as length or time of travel, gradient, existence of a cycling object, such as bike lanes, intersections, age and experience of the cyclist, and traffic volume. The vast majority of riders choose a bike because of the shortest route. In some cases, the shortest route for the rider to reach the destination means to cross the railway line or other obstacles that cyclists would rather avoid. Other articles suggest that most riders embark on a short distance trip. This modeling has sometimes been studied in conjunction with pedestrian traffic. The municipality of Novo mesto uses the GoNM system. Municipality Novo mesto (MONM) has started a bicycle rental project. This urban transport planning process often considers bicycles and pedestrians as a system of passenger network. Therefore, it is necessary to develop a methodology for estimating and analyzing bicycle rental demand. This system consists of the following stations (vertices in our network). 


\begin{tabular}{|l|l|}
\hline Code of Stations & Names of Stations \\
\hline \hline P1 & Bus Station - Topliska Road \\
P2 & BTC City Novo mesto \\
P3 & Center - Seidlova Road \\
P4 & Drska - Segova Street \\
P5 & Main Square \\
P6 & Kandijska Bridge \\
P7 & Locna - Seidlova Road \\
P8 & New Square \\
P9 & Elementary School Brsljin \\
P10 & Elementary School Smihel \\
P11 & Podbreznik \\
P12 & Ragovska Street \\
P13 & School Center Novo Mesto \\
P14 & Slavka Gruma Street \\
\hline
\end{tabular}

Table 1. List of stations of GoNM system

Using network theory, we will analyze the topological properties of different cycling networks. Using statistical methods, we will analyze the time graphs of bike rentals. The triadic census is a count of how many of the 16 possible types of triads are present in a directed graph. This model can be used to design bicycle road networks. MONM cycling research data is used to study the characteristics of the model. The model is used to study the bike rental network at MONM.

The artificial ants used in optimization with ant colonies are procedures that make up solutions using probabilities. In the gradual (step-by-step) assembly of the solution, the ant takes into account the heuristic information about the problem it solves and about pheromone trails that change during the course of the solution and that express, up to a given moment, information about the current solution to the problem. Although one ant is a very simple and primitive creature, the colony of ants is a very organized unit and very effective in solving complex tasks, e.g. finding the shortest route between two points. A stronger concentration of pheromones in the pathway means that other ants are more likely to follow this path. Figure 1 represents Ant System Algorithm [9].

The ants indirectly interact with each other through traces of the chemical substance of pheromones, which they dispose of along the way, thereby altering the environment. Pheromones evaporate over time, but the intensity on shorter paths nevertheless increases. Ants that choose the shorter route get to the food earlier and return faster, so they can drop the trail several times in the same time interval. Figure 1 shows ants in real space. Initially, we have two ants that start their journey and do the same probability (0.5) deciding between paths. The ant that chose the shorter path came faster to the finish line. The ant that chose the shorter path returns to the nest and leaves a trail of pheromones behind. The next ant detects pheromones and chooses a shorter path. After a few repetitions, more 


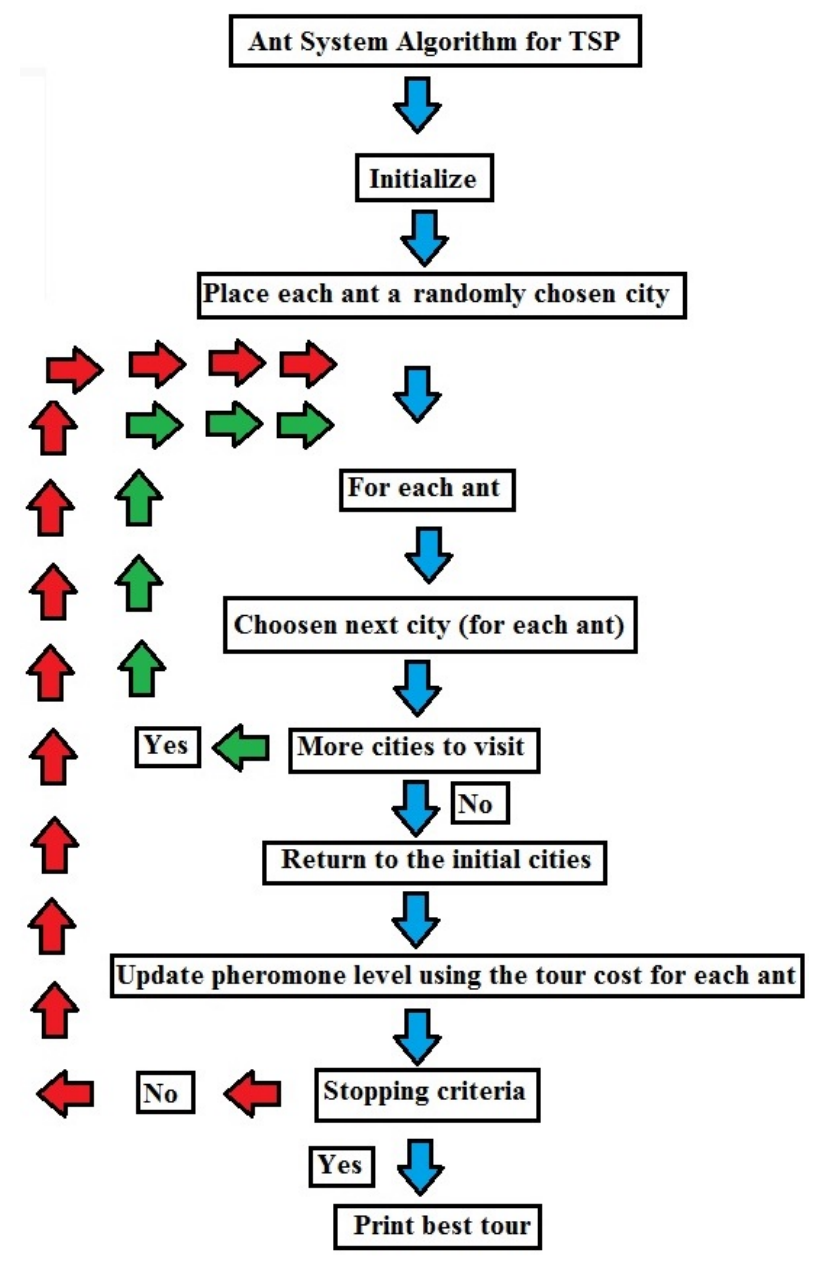

Figure 1. Ant system algorithm

and more ants are taking the path with a higher concentration of pheromones and further enhancing it. After a while, only a shorter route becomes operational.

\section{RESULTS AND DISCUSSION}

\subsection{Exploratory Network Data Analysis}

In 2019, there were 14 stations (Figure 2) where a bike could be borrowed.

Data on bicycle use were obtained by the City of Novo mesto and cover the total real loan from the introduction of the system in April 2019 to the end of November 


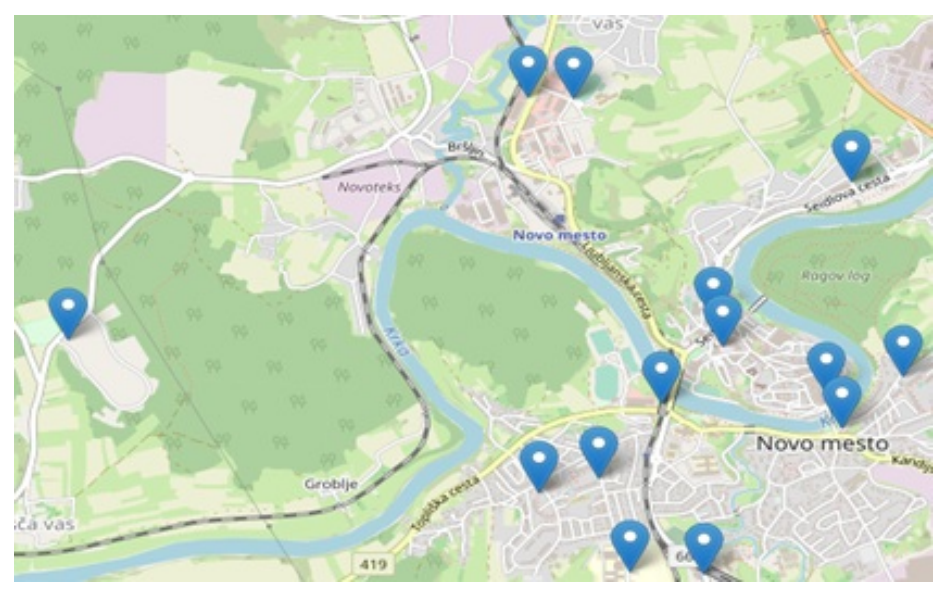

Figure 2. Micro locations of 14 GoNM stations in the city of Novo mesto in 2019

2019. In a directed network there are sixteen possible triads. This routine counts the number of each type of triad present in a directed network. The triads are labelled abcZ where $a$ is the number of reciprocated ties, $b$ is the number of unreciprocated ties and $c$ is the number of null ties. The $Z$ term is a letter $(U, C, D$ or $T)$ used to differentiate between different triads in which these numbers are the same. For an undirected network there are only 4 possible triads, namely 003, 102, 201 and 300. Table 2 presents the topological properties of the triad types of bike sharing network, which were calculated using the Pajek program [10].

\begin{tabular}{|l|r|}
\hline Triad Type & Number of Triads \\
\hline \hline type 1 triad -003 & 2 \\
type 2 triad -012 & 17 \\
type 3 triad -102 & 35 \\
type 4 triad $-021 \mathrm{D}$ & 9 \\
triad type $5-021 \mathrm{U}$ & 4 \\
type 6 triad $-021 \mathrm{C}$ & 12 \\
type 7 triad $-111 \mathrm{D}$ & 30 \\
triad of type $8-111 \mathrm{U}$ & 36 \\
triad type $9-030 \mathrm{~T}$ & 10 \\
triad type $10-030 \mathrm{C}$ & 1 \\
type $11-201$ triad & 39 \\
triad type $12-120 \mathrm{D}$ & 7 \\
triad type $13-120 \mathrm{U}$ & 17 \\
type $14-120 \mathrm{C}$ triad & 19 \\
type $15-210$ triad & 84 \\
type triad $16-300$ & 42 \\
\hline
\end{tabular}

Table 2. Topological properties of the triad network types 


\begin{tabular}{|l|r|r|r|r|r|r|r|r|r|r|r|r|r|r|}
\hline Station & P1 & P2 & P3 & P4 & P5 & P6 & P7 & P8 & P9 & P10 & P11 & P12 & P13 & P14 \\
\hline \hline P1 & 0 & 5 & 1 & 5 & 7 & 10 & 10 & 19 & 15 & 10 & 4 & 8 & 20 & 10 \\
P2 & 6 & 6 & 2 & 24 & 8 & 8 & 445 & 18 & 17 & 15 & 12 & 5 & 8 & 20 \\
P3 & 1 & 1 & 8 & 5 & 9 & 25 & 11 & 11 & 21 & 185 & 14 & 43 & 9 & 27 \\
P4 & 15 & 4 & 5 & 17 & 10 & 5 & 9 & 10 & 10 & 16 & 10 & 39 & 42 & 16 \\
P5 & 12 & 1 & 10 & 15 & 8 & 1 & 5 & 5 & 18 & 24 & 30 & 25 & 37 & 32 \\
P6 & 8 & 1 & 11 & 5 & 1 & 13 & 18 & 21 & 31 & 35 & 21 & 18 & 35 & 25 \\
P7 & 7 & 14 & 2 & 8 & 1 & 15 & 10 & 31 & 1 & 32 & 17 & 15 & 25 & 35 \\
P8 & 6 & 12 & 3 & 7 & 10 & 5 & 19 & 40 & 14 & 5 & 5 & 14 & 18 & 28 \\
P9 & 9 & 1 & 1 & 23 & 24 & 2 & 10 & 31 & 20 & 7 & 31 & 5 & 10 & 16 \\
P10 & 19 & 8 & 5 & 25 & 17 & 9 & 45 & 35 & 5 & 10 & 7 & 3 & 14 & 8 \\
P11 & 8 & 18 & 10 & 10 & 10 & 16 & 12 & 4 & 4 & 9 & 8 & 20 & 8 & 9 \\
P12 & 4 & 16 & 7 & 5 & 5 & 29 & 15 & 5 & 2 & 18 & 5 & 10 & 7 & 5 \\
P13 & 3 & 1 & 4 & 1 & 27 & 4 & 35 & 25 & 25 & 15 & 10 & 6 & 12 & 13 \\
P14 & 2 & 5 & 3 & 6 & 2 & 17 & 16 & 15 & 9 & 42 & 9 & 9 & 38 & 10 \\
\hline
\end{tabular}

Table 3. Bicycle rental and return network by 14 different GoNM stations for all days

Table 2 shows the different types of bicycle rental network triads. With the increasing bicycle stations and increased popularity of using bicycle it is expected deeper understanding of the public bicycle tranport by using concepts of triad census network analysis such as sub-graph analysis. A triadic census provides a method to detect substructure in the public bicycle network, more specifically the tendency for certain types of triadic ties. Most triads have type 15, 84, and least type 10, 1. Triads of types $9,12,13,16$ are transitive, triads of types $6,7,8,10,11,14,15$ are non-transitive and type $1,2,3,4,5$ triads do not contain links to satisfy the transitivity conditions. There are thus 76 transitive triads, 221 non-transitive triads and 67 triad types in the bicycle rental network that do not qualify for transitivity. As you can see, we have the most non-transitive triads in the bike rental network. Table 3 shows the bicycle rental and return network by 14 different locations for all days in July. Table 4 presents the statistical characteristics of bicycle rentals for each day.

\begin{tabular}{|l|r|r|r|}
\hline Day & Average (h:min:sec) & Minimum (h:min:sec) & Maximum (h:min:sec) \\
\hline \hline Monday & $0: 38: 42$ & $0: 00: 21$ & $19: 41: 47$ \\
Tuesday & $0: 39: 49$ & $0: 00: 19$ & $21: 59: 28$ \\
Wednesday & $0: 32: 51$ & $0: 00: 19$ & $11: 18: 32$ \\
Thursday & $0: 35: 58$ & $0: 00: 20$ & $21: 32: 04$ \\
Friday & $0: 48: 46$ & $0: 00: 18$ & $23: 47: 14$ \\
Saturday & $0: 51: 05$ & $0: 00: 01$ & $23: 52: 12$ \\
Sunday & $0: 57: 25$ & $0: 00: 24$ & $23: 53: 45$ \\
\hline
\end{tabular}

Table 4. Statistical characteristics of bicycle time rentals for each day in a week

Table 5 represents basic statistical characteristics, average, maximum, minimum, standard deviation and median calculation. The highest average bike rental 
is on Friday, 92.78 bicycles, and the lowest on Sunday 41.85. Most bicycle rentals are at the Main Square station on Tuesday and the lowest on Saturday at the Šmihel - Šmihel elementary school. The standard bicycle rental deviation ranges from 17.35 to 36.63 . The median reaches values between 44 and 97.5 . It is interesting to note that the short-distance bicycle rental (Main Square, Kandija Bridge and Ragovska Street) is very high. This means that bike rentals are high on short distances. We can conclude that the reason for this is the urban environment. Table 3 presents the number of bicycles borrowed in 2019 for each day of the week. Somehow the expected result is that bike rentals days are Saturday and Sunday, when no business day is the minimum. On Friday, we have the most bike rentals, 1327, and on Sunday, the least, 596. The total bike rental for 2019 is 7380 bicycles. Table 4 presents the statistical characteristics of bicycle rentals for each day. The maximum average bike rental time on Sunday and Saturday is about the same time, around one hour. It is significantly smaller during working hours. The minimum bike rental time ranges from $1 \mathrm{~min}$ on Saturday to $24 \mathrm{~min}$ on Sunday. The maximum rental time is Sunday approximately one day, specifically 23:53:45

\begin{tabular}{|l|r|r|r|r|r|r|r}
\hline Station & Monday & Tuesday & Wednesday & Thursday & Friday & Saturday & Sunday \\
\hline \hline P1 & 122 & 111 & 110 & 128 & 100 & 40 & 30 \\
P2 & 85 & 88 & 101 & 93 & 110 & 57 & 39 \\
P3 & 79 & 85 & 84 & 82 & 106 & 27 & 25 \\
P4 & 85 & 79 & 87 & 90 & 107 & 65 & 48 \\
P5 & 117 & 144 & 142 & 127 & 157 & 97 & 45 \\
P6 & 69 & 52 & 76 & 57 & 79 & 57 & 43 \\
P7 & 65 & 67 & 76 & 72 & 83 & 38 & 49 \\
P8 & 93 & 116 & 124 & 107 & 128 & 52 & 41 \\
P9 & 47 & 71 & 51 & 50 & 55 & 26 & 23 \\
P10 & 21 & 29 & 24 & 25 & 25 & 4 & 11 \\
P11 & 51 & 28 & 35 & 48 & 41 & 35 & 47 \\
P12 & 92 & 92 & 87 & 84 & 75 & 48 & 45 \\
P13 & 105 & 117 & 140 & 114 & 138 & 65 & 85 \\
P14 & 83 & 103 & 90 & 104 & 95 & 48 & 55 \\
\hline sum & 1114 & 1182 & 1227 & 1181 & 1299 & 659 & 586 \\
\hline mean & 79.57 & 84.42 & 87.64 & 84.35 & 92.78 & 47.07 & 41.85 \\
max & 122 & 144 & 142 & 128 & 157 & 97 & 85 \\
min & 21 & 12 & 20 & 9 & 25 & 4 & 7 \\
sd & 26.57 & 32.24 & 33.97 & 29.93 & 35.30 & 21.28 & 16.72 \\
median & 84 & 86.5 & 87 & 87 & 97.5 & 48 & 44 \\
\hline
\end{tabular}

Table 5. Number of bicycles borrowed at each station for each day during March-November 2019 and basic statistical characteristics

Table 6 presents description of GoNM stations with different parameters for which we expect to have impact on the relevance of each station: the proximity to cycling lane (YES, if cycling lane is very close, NO otherwise), proximity to resi- 
dential neighbourhood, proximity to shopping centre, distance to shopping centre, proximity to public buildings, distance to public buildings, distance to closest GoNM station, number of working places in the proximity, and finally, the last column contains the numbers of bike rentals in the observed period.

\begin{tabular}{|c|c|c|c|c|c|c|c|c|c|}
\hline $\begin{array}{l}\text { Station } \\
a\end{array}$ & $\begin{array}{l}\text { Prox. to } \\
\text { Cyc. Lane }\end{array}$ & $\begin{array}{l}\text { Prox. to } \\
\text { Resid. }\end{array}$ & $\begin{array}{l}\text { Prox. to } \\
\text { Shopping }\end{array}$ & $\begin{array}{r}\text { Dist. to } \\
\text { Shopping }\end{array}$ & $\begin{array}{c}\text { Prox. Public } \\
\text { Houses }\end{array}$ & $\begin{array}{l}\text { Dist. to } \\
\text { Pub. h. }\end{array}$ & $\begin{array}{l}\text { Dist. to } \\
\text { GoNM }\end{array}$ & $\begin{array}{l}\text { \# Working } \\
\text { Places in Neighb. }\end{array}$ & $\begin{array}{r}\text { Total } \\
\text { Rentals }\end{array}$ \\
\hline$\overline{\mathrm{P} 1}$ & $\overline{\mathrm{YES}}$ & $\overline{\mathrm{YES}}$ & $\overline{\text { YES }}$ & 76 & YES & 240 & 600 & "many & $\overline{641}$ \\
\hline P2 & YES & $\mathrm{NO}$ & YES & 5 & YES & 100 & 300 & many & 573 \\
\hline P3 & YES & $\mathrm{NO}$ & $\mathrm{NO}$ & 700 & YES & 110 & 300 & many & 488 \\
\hline $\mathrm{P} 4$ & $\mathrm{NO}$ & YES & $\mathrm{NO}$ & 700 & $\mathrm{NO}$ & 800 & 400 & medium & 561 \\
\hline P5 & YES & $\mathrm{NO}$ & $\mathrm{NO}$ & 1800 & YES & 96 & 230 & medium & 829 \\
\hline $\mathrm{P} 6$ & YES & YES & $\mathrm{NO}$ & 1500 & YES & 140 & 230 & few & 433 \\
\hline P7 & YES & YES & $\mathrm{NO}$ & 2000 & YES & 290 & 1000 & few & 450 \\
\hline P8 & YES & $\mathrm{NO}$ & YES & 50 & YES & 100 & 300 & many & 661 \\
\hline P9 & $\mathrm{NO}$ & YES & YES & 300 & YES & 100 & 300 & medium & 323 \\
\hline P10 & $\mathrm{NO}$ & YES & $\mathrm{NO}$ & 800 & YES & 140 & 750 & medium & 139 \\
\hline P11 & $\mathrm{NO}$ & YES & $\mathrm{NO}$ & 2000 & $\mathrm{NO}$ & 3000 & 3000 & medium & 285 \\
\hline P12 & $\mathrm{NO}$ & YES & $\mathrm{NO}$ & 2200 & YES & 300 & 400 & few & 523 \\
\hline $\mathrm{P} 13$ & $\mathrm{NO}$ & YES & $\mathrm{NO}$ & 1000 & YES & 10 & 600 & many & 764 \\
\hline P14 & YES & YES & YES & 30 & $\mathrm{NO}$ & 800 & 400 & medium & 578 \\
\hline
\end{tabular}

Table 6. Parameters that may affect the total number of bike rental at GoNM stations

We wanted to know in where to put the additional 6 stations at the request of the Novo mesto municipality. Table 6 shows the parameters that affect bicycle rental according to economic and demographic criteria. These parameters are the proximity to the bike lane, proximity to stand neighborhoods, total number of bike rentals in 2019, proximity to mall, distance to mall, proximity to public buildings, distance to public buildings, and distance to the nearest GoNM station. The penultimate column represents the decisive YES/NO and the last one represents the distance (the sum of the distances relative to the other distances in the table). Figure 3 presents the planned new stations between the marked stations, which are marked red. We were interested in how the cycle path affects the placement of additional stations.

Table 7 presents the impact of the bike lane on the placement of new stations between the existing stations. By 1 we have marked that the bike lane is near the station; by 0 that it is not; and by 0.5 that the bike lane is on the part of the route between the two stations.

We use ACO settings: population size 30, evaporation rate 0.2 , deposition rate 0.3 , parameter alpha 1 and parameter beta 2 .

\subsection{Regression Tree Explanation of Total Number of Rentals}

In this subsection we present results of regression tree analysis, which indicates the factors relevant for the total number of rentals at each GoNM station. Figure 7 shows that the most visited GoNM stations (the stations with higher total number of rentals in the observed period) are those which have 


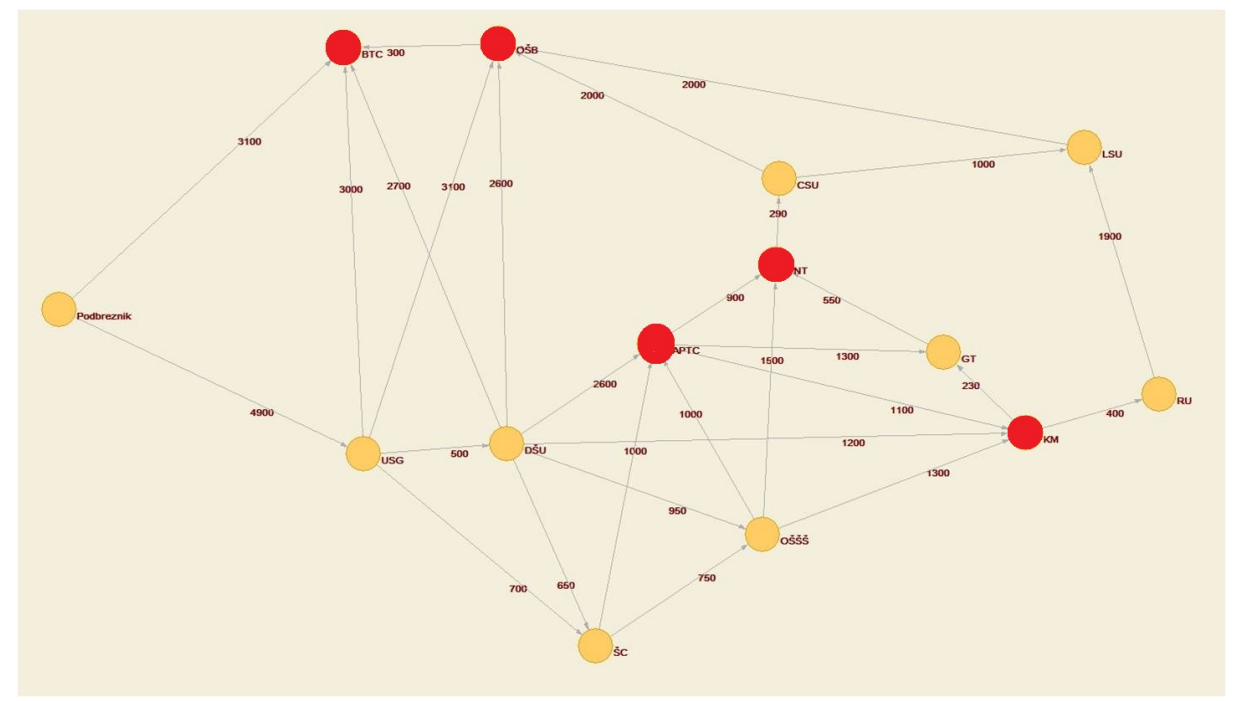

Figure 3. New stations foreseen between the marked stations, which are marked in red according to Table 6

- distance to the closest GoNM station less than $675 \mathrm{~m}$,

- distance to the closest public building less than $98 \mathrm{~m}$.

On the other hand, stations with the distance to the closest GoNM station larger than $675 \mathrm{~m}$ or with distance to the closest shopping center larger than approx. $190 \mathrm{~m}$ have the lowest number of rentals.

\begin{tabular}{|l|l|l|l|l|l|l|l|l|l|l|l|l|l|l|}
\hline Station & P1 & P2 & P3 & P4 & P5 & P6 & P7 & P8 & P9 & P10 & P11 & P12 & P13 & P14 \\
\hline \hline P1 & 0 & 0.5 & 1 & 0 & 0.5 & 0 & 0 & 0 & 0.5 & 0 & 0.5 & 0 & 0 & 0 \\
P2 & 0.5 & 0 & 0.5 & 0 & 0 & 0 & 0.5 & 1 & 1 & 0.5 & 1 & 0.5 & 0 & 0 \\
P3 & 0 & 1 & 0 & 0.5 & 0.5 & 0.5 & 1 & 1 & 1 & 0.5 & 1 & 0.5 & 0.5 & 0.5 \\
P4 & 0 & 0 & 0.5 & 0 & 0 & 0 & 0 & 0 & 0.5 & 0.5 & 0 & 0 & 0 & 0 \\
P5 & 0 & 1 & 1 & 0.5 & 0 & 1 & 0.5 & 0.5 & 1 & 0.5 & 0 & 0.5 & 0.5 & 0.5 \\
P6 & 0 & 1 & 1 & 0.5 & 1 & 0 & 1 & 1 & 1 & 0.5 & 0 & 0.5 & 0.5 & 0.5 \\
P7 & 0 & 1 & 1 & 0.5 & 0.5 & 0.5 & 0 & 1 & 1 & 0.5 & 1 & 0.5 & 0.5 & 0.5 \\
P8 & 0 & 1 & 1 & 0.5 & 0 & 0.5 & 1 & 0 & 1 & 0.5 & 0.5 & 0.5 & 0.5 & 0.5 \\
P9 & 0.5 & 1 & 1 & 0 & 0 & 0 & 0 & 1 & 0 & 0.5 & 1 & 0.5 & 0 & 0 \\
P10 & 0 & 0.5 & 0.5 & 0.5 & 0.5 & 0.5 & 0.5 & 0.5 & 0.5 & 0 & 0 & 0.5 & 0.5 & 0.5 \\
P11 & 0.5 & 0 & 0 & 0 & 0 & 0 & 0 & 0 & 0 & 0 & 0 & 0 & 0 & 0 \\
P12 & 0 & 0.5 & 0.5 & 0.5 & 0.5 & 0 & 0.5 & 0.5 & 0.5 & 0.5 & 0.5 & 0 & 0.5 & 0.5 \\
P13 & 0 & 0.5 & 0.5 & 0 & 0.5 & 0.5 & 0.5 & 0.5 & 0.5 & 0.5 & 0 & 0.5 & 0 & 0 \\
P14 & 0 & 0.5 & 0.5 & 0 & 0.5 & 0.5 & 0.5 & 0.5 & 0.5 & 0.5 & 0 & 0.5 & 0 & 0 \\
\hline
\end{tabular}

Table 7. Parameters that affect bike rental based on economic and demographic criteria 


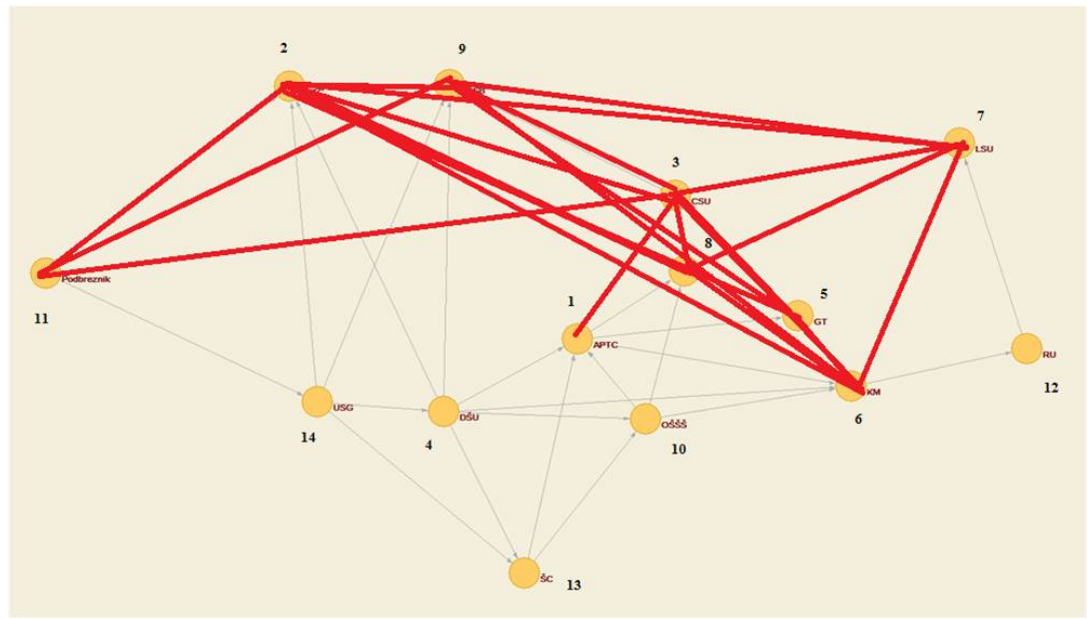

Figure 4. Connections between stations where the bike lane is located according to Table 7

The data are presented in Table 7. By 1 we denote that the cycle path is near the station; by 0 that it is not; and by 0.5 that the cycle path is on the part of the path between the two stations. Figure 4 presents the connections between stations where the cycle track is located according to Table 7. Figure 5 represents the predicted locations for data analysis. Figure 6 represents the shortest path of

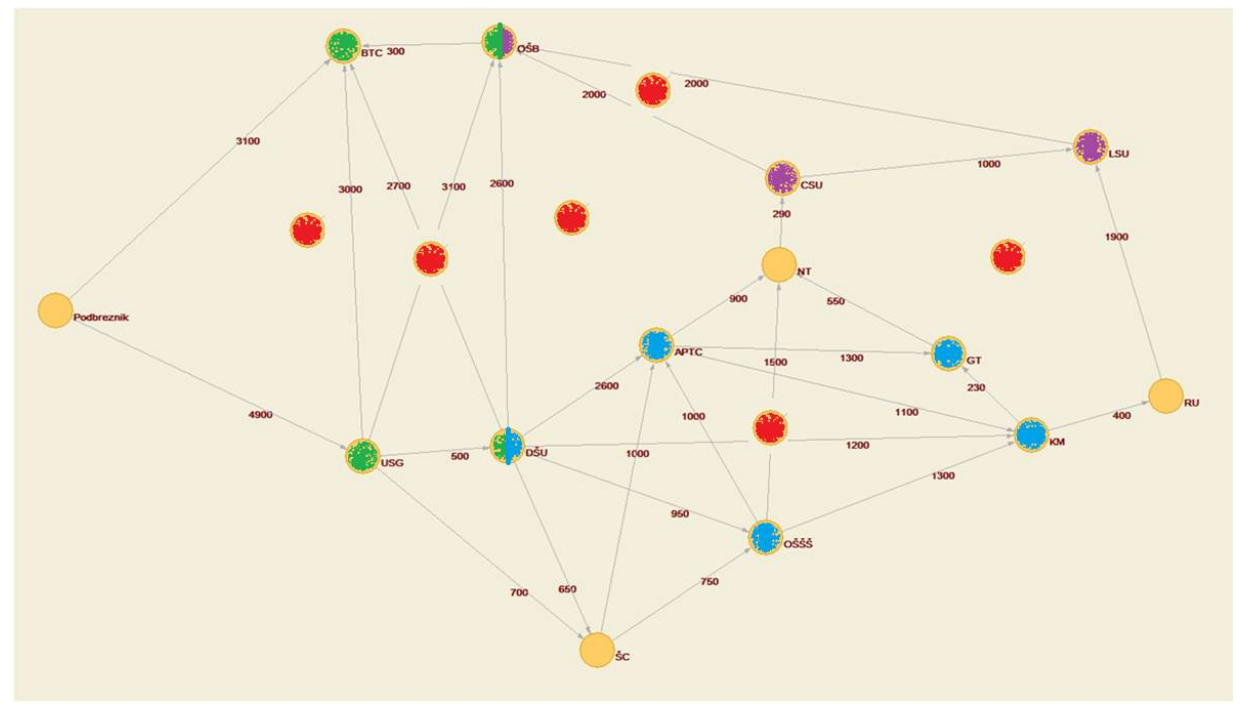

Figure 5. Estimated locations for data analysis 
Modeling Public Transport Network System by Using Statistics, Network Theory... 1171

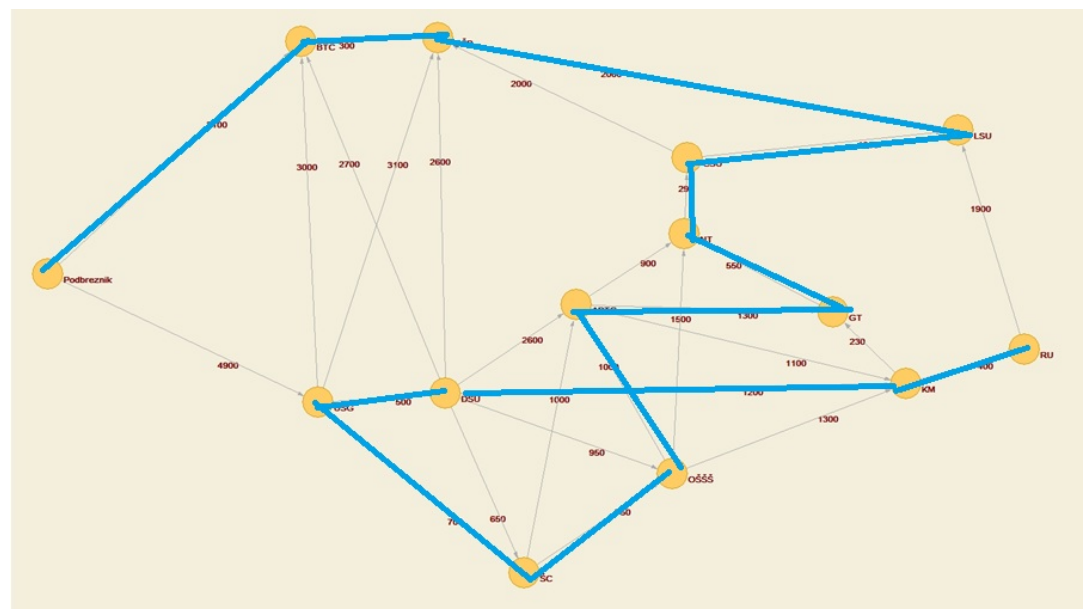

Figure 6 . The shortest way to get around 14 stops $(12190 \mathrm{~m})$

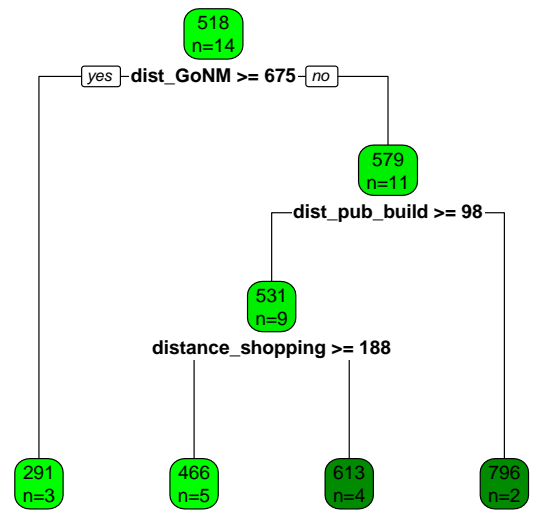

Figure 7. Regression tree explanation of the total number of rentals

bicycle development across 14 stations $(12190 \mathrm{~m})$ obtained by swarm intelligence, an ant colony.

The regression tree analysis shows that the most important factors for the number of rentals at each GoNM station are the proximity to other GoNM stations, public buildings and shopping centers.

\section{CONCLUSION}

In this article we have presented the statistical and topological characteristics of the public bicycle rental network for 2019 in Novo mesto. The use of bicycles means 
a reduction in the number of vehicles on the roads, which reduces traffic congestion, reduces driving speed (more fluid traffic) and results in a very significant reduction in atmospheric pollution, resulting in fewer respiratory diseases, less economic loss from work due to sick leave, less health care expenditures, less deterioration on planted areas and buildings, greater attractiveness of the city center due to improved quality of life in cities and reduced energy consumption. The statistical analysis of the data and the topological characteristics of the bicycle rental network can help. We shown application one of method of swarm intelligence in public transport system, especially in a bike sharing system in optimization problem. We used ACO for find shortest path. With the increase of cycling stations and the increased use of network analysis methods, it is expected that a better understanding and organization of public passenger transit could be developed using further research concepts of different types of triad network such as sub-graph analysis, distribution analysis and stability analysis.

\section{Acknowledgement}

The investment is co-financed by the Republic of Slovenia and the European Union from the European Regional Development Fund.

\section{REFERENCES}

[1] Roman, M.-Roman, M.: Bicycle Transport as an Opportunity to Develop Urban Tourism - Warsaw Example. Procedia - Social and Behavioral Sciences, Vol. 151, 2014, pp. 295-301, doi: 10.1016/j.sbspro.2014.10.027

[2] Frade, I.-Ribeiro, A.: Bicycle Sharing Systems Demand. Procedia - Social and Behavioral Sciences, Vol. 111, 2014, pp. 518-527, doi: 10.1016/j.sbspro.2014.01.085.

[3] Anderson, M.: Mobile Technology and Home Broadband 2019. Pew Research Center, 2019. Available at: https://www.pewresearch.org/internet/2019/06/ 13/mobile-technology-and-home-broadband-2019/.

[4] Deakin, M.: From Intelligent to Smart Cities. Chapter 2. In: Deakin, M. (Ed.): Smart Cities: Governing, Modelling and Analysing the Transition. Taylor and Francis, 2014.

[5] Illowski, B.-Dean, S. L.: Introductory Statistics. OpenStax College, Rice University, 2013.

[6] Korczynski, W.-Byrski, A.-DȩBski, R.-Kisiel-Dorohinicki, M.: Classic and Agent-Based Evolutionary Heuristics for Shape Optimization of Rotating Discs. Computing and Informatics, Vol. 36, 2017, No. 2, pp. 331-352, doi: 10.4149/cai_2017_2_331

[7] Kumar, A.-Kumar, R.-Sharma, A.: Energy Aware Resource Allocation for Clouds Using Two Level Ant Colony Optimization. Computing and Informatics, Vol. 37, 2018, No. 1, pp. 76-108, doi: 10.4149/cai_2018_1_76. 
[8] Buehler, R.-Pucher, J.: Sustainable Transport in Freiburg: Lessons from Germany's Environmental Capital. International Journal of Sustainable Transportation, Vol. 5, 2011, No. 1, pp. 43-70, doi: 10.1080/15568311003650531.

[9] Red Apple Tutorials: Traveling Salesman Problem Using Ant Colony Optimization. Video Presentation. https://www.youtube.com/watch?v=9KVg3gv_0cA.

[10] De Nooy, W.-Mrvar, A.-Batagelu, V.: Exploratory Social Network Analysis with Pajek. Second Edition. Cambridge University Press, 2011.

Matej BABIČ received his Ph.D. degree in computer science from the Faculty of Electrical Engineering and Computer Science of the University of Maribor, Slovenia. He studied mathematics at the Faculty of Education in Maribor. He is Assistant Professor at the Faculty of Information Studies, Novo mesto, Slovenia. His research interest is in fractal geometry, graph theory, network, intelligent systems, hybrid machine learning, topography of materials after hardening and public transport systems.

Ladislav HuUChÝ is the Vice-Director of the Institute of Informatics of the Slovak Academy of Sciences and also the Head of the Department of Parallel and Distributed Computing at the Institute. He received his M.Sc. and Ph.D. degrees, both in computer science. He is R \& D Project Manager, Work Package Leader in a number of 4FP, $5 \mathrm{FP}$ and $6 \mathrm{FP}$ projects, as well as in Slovak R\&D projects (VEGA, APVT, SPVV). He is a member of IEEE, ERCIM, SRCIM, and EuroMicro consortiums, the Editor-in-Chief of the Journal Computing and Informatics. He is also (co-)author of scientific books and numerous scientific papers, contributions and invited lectures at international scientific conferences and workshops. He also gives lectures at the Slovak University of Technology in Bratislava, Slovakia, and he is Supervisor and Consultant for Ph.D., Master's and Bachelor's studies.

Branko ŠTER received his B.Sc. and M.Sc. degrees in electrical engineering and his Ph.D. degree in computer science from the University of Ljubljana, Slovenia, in 1993, 1996 and 1999, respectively. He works at the Faculty of Computer and Information Science, University of Ljubljana, as Professor. His main research interests include machine learning and hardware implementations of neural networks.

Janez PovH is Senior Researcher at the Faculty of Mechanical Engineering, University of Ljubljana. He received his Ph.D. in 2006 on topic application of semidefinite and copositive programming and combinatorial optimization at the Faculty of Mathematics and Physics, University of Ljubljana. His research focus is development of new methods in mathematical optimization, based on the intensive use of supercomputers. He is Project Leader of several industrial, European and national scientific projects in the domain of mathematical optimization, high-performance computing and big data analysis. 